\title{
LIFTING-BASED LAPLACIAN PYRAMID RECONSTRUCTION SCHEMES
}

\author{
Lijie $\operatorname{Liu}^{\dagger}$, Lu Gan ${ }^{\ddagger}$ and Trac D. Tran ${ }^{\dagger}$ \\ ${ }^{\dagger}$ Department of Electrical and Computer Engineering \\ The Johns Hopkins University, Baltimore, MD 21218, USA \\ ${ }^{\ddagger}$ School of Engineering and Design \\ Brunel University, Uxbridge, West London, UK
}

\begin{abstract}
Laplacian Pyramid (LP) provides a redundant signal representation and can be characterized as an oversampled filter bank (FB). In this paper, a generic lifting-based parameterization reconstruction algorithm is proposed to characterize all LP synthesis banks that can satisfy the perfect reconstruction property. Two typical lifting-based LP reconstruction schemes are then derived from this general representation. The first scheme presents the dual frame LP reconstruction and its closed-form solutions for any LP filters. The second LP reconstruction scheme leads to an efficient FB, which demonstrates improvements over the usual LP reconstruction in the presence of noise.
\end{abstract}

Index Terms - Laplacian pyramid, lifting, oversampled filter bank, dual frame

\section{INTRODUCTION}

The Laplacian pyramid (LP) introduced by Burt and Adelson [1] has been proved to be a useful tool in image/video processing and communication [2]. For example, LP has been the basic module to support spatial scalability in video coding standards such as H.264 SVC standard [3]. The LP provides an overcomplete representation of visual signals, which can capture salient features of signals at different resolutions. It is an implicit oversampling system, and can be characterized as an oversampled filter bank (FB) or frame [4]. As the inverse of an oversampled analysis FB, the LP reconstruction should then have infinite realizations that can satisfy perfect reconstruction (PR) property [5]. From the frame theory, Do and Vetterli presented a complete parameterization of all synthesis FBs that can yield PR for a given LP decomposition with decimation factor $M$ [4]. Such general LP reconstruction has $M^{2}+M$ free entries. Moreover, they revealed that the traditional LP reconstruction is suboptimal, and proposed an efficient frame-based LP reconstruction scheme. This scheme requires the LP filters to be biorthogonal in order to achieve PR [4]. While a biorthogonal filter may introduce significant aliasing in the downsampled coarse LP signal. Thus, it may not be advisable for some applications such as spatially scalable video coding [6]. To overcome the biorthogonality limitation, a method called lifted pyramid was presented to improve scalable video coding efficiency in [6]. The lifted pyramid introduced an additional lifting step into the LP decomposition so that the PR condition can be satisfied. However, this modification in the LP decomposition causes the lifted pyramid has more significant high-frequency components thus requires larger bit rate. Therefore, it is still undesirable in the context of scalable video compression.

In this paper, we revisit the FB representation for a given LP decomposition, and present a generic lifting-based FB representation.
We offer a complete lifting-based parameterization of all LP synthesis FBs, and derive two typical LP reconstruction schemes. Both schemes are shown to perform better than the usual LP reconstruction method.

This paper is organized as follows. Section 2 reviews the LP scheme and its FB representation. Section 3 presents the generic lifting-based representation of the LP scheme. As an important typical case, the close-form solutions of dual frame for the LP reconstruction are given in Section 4. Because the dual frame reconstruction usually invokes IIR filters, Section 5 studies another typical lifting-based LP reconstruction scheme with an efficient FB, which is demonstrated to have better performance than the usual LP reconstruction. Finally, conclusions are drawn in Section 6.

\section{LAPLACIAN PYRAMID AND OVERSAMPLED FILTER BANK}

The LP decomposition and its usual reconstruction can be illustrated in Fig 1, where $H(z)$ and $G(z)$ are the decimation and interpolation filters, respectively. In the LP decomposition, the coarse approximation $c[n]$ of an input signal $x[n]$ is generated through $H(z)$ filtering followed by downsampling. Then, $c[n]$ is upsampled and filtered to provide a prediction signal to generate the prediction error signal $d[n]$. In the usual LP reconstruction, the reconstruction signal $\hat{x}[n]$ is obtained by simply adding $d[n]$ back to the prediction from $c[n]$. Since $c[n]$ and $d[n]$ have more coefficients than $x[n]$, the LP is an overcomplete system.

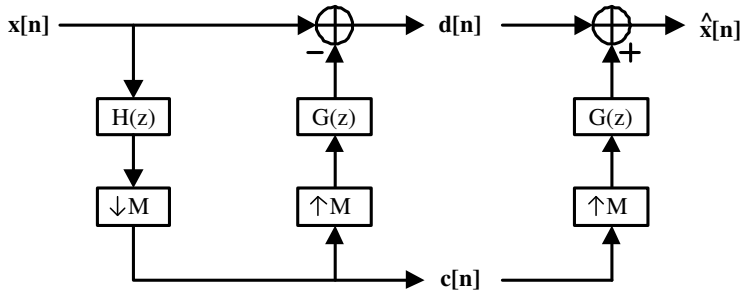

Fig. 1. The LP decomposition (left part) and its usual reconstruction (right part).

The LP realizes a frame expansion as $x[n]$ can be always reconstructed from $c[n]$ and $d[n]$ [4]. In the FB point of view, the LP can be formulated as an $M+1$-channel oversampled FB with a sampling factor $M$ [4]. Let superscript $H$ denote the Hermitian transpose. The polyphase analysis matrix for the LP decomposition in Fig. 1 is

$$
\mathbf{E}(z)=\left[\begin{array}{c}
\mathbf{h}(z) \\
\mathbf{I}_{M}-\mathbf{g}^{H}(z) \mathbf{h}(z)
\end{array}\right]
$$


where $1 \times M$ vectors $\mathbf{h}(z)$ and $\mathbf{g}(z)$ are Type-I polyphase matrices of $H(z)$ and $G(z)$, respectively. The corresponding polyphase synthesis matrix of the usual LP reconstruction scheme is

$$
\mathbf{R}(z)=\left[\begin{array}{ll}
\mathbf{g}^{H}(z) & \mathbf{I}_{M}
\end{array}\right] .
$$

For any $H(z)$ and $G(z),(1)$ and (2) satisfy the PR condition, i.e.,

$$
\mathbf{R}(z) \mathbf{E}(z)=\mathbf{I}_{M} .
$$

While the LP reconstruction (2) is suboptimal as explained in [4].

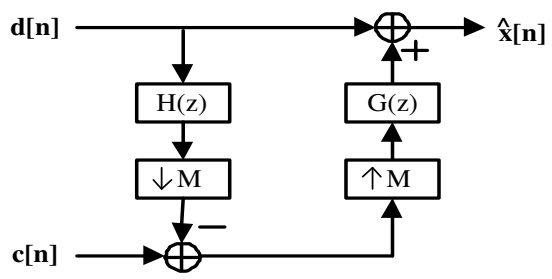

Fig. 2. The frame-based reconstruction scheme for the LP.

As illustrated in Fig. 2, the frame-based LP reconstruction scheme proposed in [4] has the polyphase synthesis matrix as

$$
\mathbf{R}(z)=\left[\begin{array}{ll}
\mathbf{g}^{H}(z) & \mathbf{I}_{M}-\mathbf{g}^{H}(z) \mathbf{h}(z)
\end{array}\right] .
$$

The PR condition (3) is satisfied only when $H(z)$ and $G(z)$ are biorthogonal filters, i.e., $\mathbf{h}(z) \mathbf{g}^{H}(z)=1$. And the reconstruction (4) leads an improvement over the traditional reconstruction (2) when $H(z)$ and $G(z)$ are orthogonal or near orthogonal filters. Under this restriction, $\mathbf{E}(z)$ is a paraunitary matrix, and (4) becomes the dual frame of (1).

\section{GENERIC LIFTING-BASED RECONSTRUCTION}

For any LP filters $H(z)$ and $G(z)$, the PR condition (3) can be always satisfied. In [4], a general complete parameterization of all PR synthesis FBs is formulated as

$$
\mathbf{R}(z)=\tilde{\mathbf{R}}(z)+\mathbf{U}(z)\left[\mathbf{I}_{M+1}-\mathbf{E}(z) \tilde{\mathbf{R}}(z)\right],
$$

where $\tilde{\mathbf{R}}(z)$ can be any particular left inverse of $\mathbf{E}(z)$, and $\mathbf{U}(z)$ is an $M \times(M+1)$ matrix with bounded entries. (5) thus has $M^{2}+M$ degrees of design freedom. However, the number of free parameters can be further reduced based on the following lifting parameterization.

For any LP filters, (1) can be factorized into two lifting steps as

$$
\mathbf{E}(z)=\left[\begin{array}{cc}
1 & \mathbf{0}_{1 \times M} \\
-\mathbf{g}^{H}(z) & \mathbf{I}_{M}
\end{array}\right]\left[\begin{array}{cc}
1 & \mathbf{h}(z) \\
\mathbf{0}_{M \times 1} & \mathbf{I}_{M}
\end{array}\right]\left[\begin{array}{c}
\mathbf{0}_{1 \times M} \\
\mathbf{I}_{M}
\end{array}\right] .
$$

To invert a lifting step, we can simply subtract out what has been added in at the forward transform. Thus, the left inverse of $\mathbf{E}(z)$ can be easily achieved by inverting the lifting steps in (6). We then can obtain the new generic form of $\mathbf{R}(z)$.

Theorem 1. For a given LP, its synthesis polyphase matrix $\mathbf{R}(z)$ has the general lifting-based representation as

$$
\begin{aligned}
\mathbf{R}(z) & =\left[\begin{array}{ll}
\mathbf{0}_{M \times 1} & \mathbf{I}_{M}
\end{array}\right]\left[\begin{array}{cc}
1 & \mathbf{0}_{1 \times M} \\
\mathbf{p}^{H}(z) & \mathbf{I}_{M}
\end{array}\right] \\
& \times\left[\begin{array}{cc}
1 & -\mathbf{h}(z) \\
\mathbf{0}_{M \times 1} & \mathbf{I}_{M}
\end{array}\right]\left[\begin{array}{cc}
1 & \mathbf{0}_{1 \times M} \\
\mathbf{g}^{H}(z) & \mathbf{I}_{M}
\end{array}\right] \\
& =\left[\begin{array}{ll}
\mathbf{p}^{H}(z) & \mathbf{I}_{M}
\end{array}\right]\left[\begin{array}{cc}
1-\mathbf{h}(z) \mathbf{g}^{H}(z) & -\mathbf{h}(z) \\
\mathbf{g}^{H}(z) & \mathbf{I}_{M}
\end{array}\right],
\end{aligned}
$$

where $\mathbf{p}(z)$ is an arbitrary $1 \times M$ vector with bounded entries.

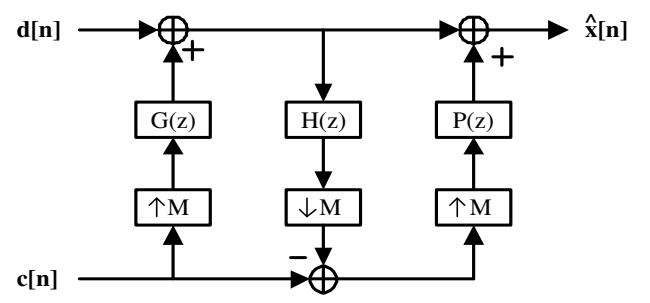

Fig. 3. Generic lifting-based LP reconstruction scheme. Given $H(z)$ and $G(z)$, it leads to perfect reconstruction for any $P(z)$.

Let $\mathbf{p}(z)$ be the type-I polyphase vector of a filter $P(z)$. Then, the reconstruction matrix (7) can be demonstrated in Fig. 3. For a given LP decomposition, (7) only has $M$ degrees of design freedom. Despite the number of free parameters reduction, (7) covers the same design space of the synthesis FBs as (5). Specifically, we can directly obtain the following two special cases from (7):

Case 1: If $\mathbf{p}(z)=\mathbf{0}_{1 \times M}$, the synthesis matrix (7) becomes

$$
\begin{aligned}
\mathbf{R}(z) & =\left[\begin{array}{ll}
\mathbf{0}_{M \times 1} & \mathbf{I}_{M}
\end{array}\right]\left[\begin{array}{cc}
1 & -\mathbf{h}(z) \\
\mathbf{0}_{1 \times M} & \mathbf{I}_{M}
\end{array}\right]\left[\begin{array}{cc}
1 & \mathbf{0}_{M \times 1} \\
\mathbf{g}^{H}(z) & \mathbf{I}_{M}
\end{array}\right] \\
& =\left[\begin{array}{ll}
\mathbf{g}^{H}(z) & \mathbf{I}_{M}
\end{array}\right],
\end{aligned}
$$

which is just the usual LP reconstruction matrix (2).

Case 2: If $H(z)$ and $G(z)$ are biorthogonal filters, then $\mathbf{h}(z) \mathbf{g}^{H}(z)$ $=1$. Let $P(z)=G(z)$, i.e., $\mathbf{p}(z)=\mathbf{g}(z)$. (7) can be rewritten as

$$
\begin{aligned}
\mathbf{R}(z) & =\left[\begin{array}{ll}
\mathbf{g}^{H}(z) & \mathbf{I}_{M}
\end{array}\right]\left[\begin{array}{cc}
1 & -\mathbf{h}(z) \\
\mathbf{0}_{M \times 1} & \mathbf{I}_{M}
\end{array}\right]\left[\begin{array}{cc}
1 & \mathbf{0}_{1 \times} \\
\mathbf{g}^{H}(z) & \mathbf{I}_{M}
\end{array}\right] \\
& =\left[\begin{array}{ll}
\mathbf{g}^{H}(z) & \mathbf{I}_{M}-\mathbf{g}^{H}(z) \mathbf{h}(z)
\end{array}\right]
\end{aligned}
$$

which is the frame-based pyramid reconstruction (4) proposed in [4].

\section{DUAL FRAME RECONSTRUCTION}

For any filters $H(z)$ and $G(z)$, the reconstruction synthesis matrix (7) can have certain desired properties by optimizing $\mathbf{p}(z)$. A common question is that how we should choose $\mathbf{p}(z)$ such that (7) can minimize the reconstruction errors when white noise is introduced into LP coefficients. Such optimization problem is to find the dual frame reconstruction solution. Through error analysis of the LP system, we have the general close-form solutions of dual frame reconstruction as given in Theorem 2. The details derivations and proofs can be found in [7].

Theorem 2. For the LP with polyphase analysis matrix $\mathbf{E}(z)$ given in (1), its dual frame reconstruction can be expressed as

$$
\mathbf{E}^{\dagger}(z)=\left[\begin{array}{ll}
\mathbf{p}_{o p t}^{H}(z) & \mathbf{I}_{M}
\end{array}\right]\left[\begin{array}{cc}
1-\mathbf{h}(z) \mathbf{g}^{H}(z) & -\mathbf{h}(z) \\
\mathbf{g}^{H}(z) & \mathbf{I}_{M}
\end{array}\right],
$$

where $\mathbf{p}_{\text {opt }}(z)=\frac{\mathbf{h}(z)-d(z) \mathbf{g}(z)}{d(z) d^{H}(z)+\mathbf{h}(z) \mathbf{h}^{H}(z)}$ and $d(z)=1-\mathbf{h}(z) \mathbf{g}^{H}(z)$.

Remarks:

1. When $H(z)$ and $G(z)$ are biorthogonal filters, i.e., $\mathbf{h}(z) \mathbf{g}^{H}(z)=$ 1 , we can directly have the dual frame of (1) from Theorem 2 as

$$
\mathbf{E}^{\dagger}(z)=\left[\begin{array}{ll}
\mathbf{g}^{H}(z) & \mathbf{I}_{M}-\frac{\mathbf{h}^{H}(z) \mathbf{h}(z)}{\mathbf{h}(z) \mathbf{h}^{H}(z)}
\end{array}\right] .
$$




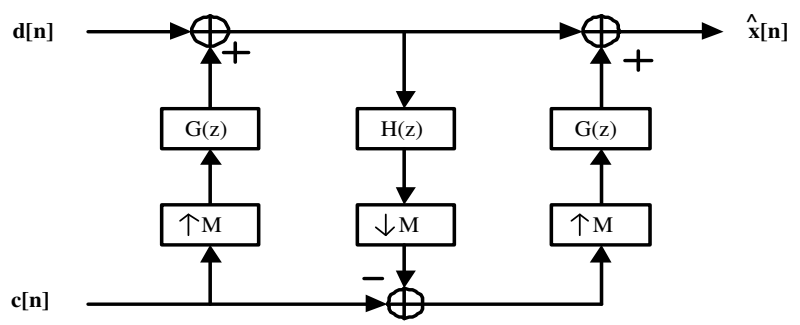

Fig. 4. A special lifting-based LP reconstruction scheme. It satisfies prefect reconstruction condition for any $H$ and $G$ filters. When $H$ and $G$ are biorthogonal, it becomes the frame-based pyramid reconstruction.

Note that the same dual frame formulation (11) for biorthogonal LP filters has been derived in [8] using the backward Greville formula. Here, (11) is obtained as a special realization of the general dual frame solution (10).

2. When $H(z)$ is an orthogonal filter and $H(z)=G(z)$, we have $\mathbf{p}(z)=\mathbf{g}(z)$. Then, (10) boils down to

$$
\mathbf{E}^{\dagger}(z)=\left[\begin{array}{ll}
\mathbf{h}^{H}(z) & \mathbf{I}_{M}-\mathbf{h}^{H}(z) \mathbf{h}(z)
\end{array}\right],
$$

which exactly equals to $\mathbf{E}^{H}(z)$ as expected.

3. Given FIR filters $H(z)$ and $G(z)$, the dual frame $\mathbf{E}^{\dagger}(z)$ given by (10) generally corresponds to an IIR FB. If $L(z)=d(z) d^{H}(z)+$ $\mathbf{h}(z) \mathbf{h}^{H}(z)$ is a positive constant, $\mathbf{E}^{\dagger}(z)$ is an FIR FB. Otherwise, we can approximate $L(z)$ by one constant to realize an FIR implementation.

\section{A SPECIAL LIFTING-BASED RECONSTRUCTION}

\subsection{New LP Reconstruction Matrix}

Considering the dual frame reconstruction (10) normally involves IIR filters, which are undesired in real applications, we present another special realization of the general lifting-based LP reconstruction (7) and let $\mathbf{p}(z) \equiv \mathbf{g}(z)$. This special LP reconstruction then has the following synthesis FB

$$
\begin{aligned}
\mathbf{R}(z) & =\left[\begin{array}{ll}
\mathbf{g}^{H}(z) & \mathbf{I}_{M}
\end{array}\right]\left[\begin{array}{cc}
1-\mathbf{h}(z) \mathbf{g}^{H}(z) & -\mathbf{h}(z) \\
\mathbf{g}^{H}(z) & \mathbf{I}_{M}
\end{array}\right] \\
& =\left[\begin{array}{ll}
\mathbf{g}^{H}(z) T(z) & \mathbf{I}_{M}-\mathbf{g}^{H}(z) \mathbf{h}(z)
\end{array}\right],
\end{aligned}
$$

where $T(z)=2-\mathbf{h}(z) \mathbf{g}^{H}(z)$. As shown in Fig. 4, it is interesting to see that this special reconstruction scheme is actually a cascade of the usual LP reconstruction (2) followed by the frame-based LP reconstruction (4). Obviously, when $H(z)$ and $G(z)$ are biorthogonal filters such that $\mathbf{h}(z) \mathbf{g}^{H}(z)=1$, then $T(z)=1$ and (12) just becomes the frame-based pyramid reconstruction (4). That's, (4) can be considered as a special case of (12). When $H(z)$ and $G(z)$ are orthogonal filters, (12) realizes the dual frame reconstruction as well.

Table 1. A 13-tap lowpass filter and its coefficients.

\begin{tabular}{|c|ccccccc|}
\hline$n$ & 0 & \pm 1 & \pm 2 & \pm 3 & \pm 4 & \pm 5 & \pm 6 \\
\hline$l[n] / \sqrt{2}$ & 26 & 19 & 5 & -3 & -4 & 0 & 2 \\
\hline
\end{tabular}

Recall that when $H(z)$ and $G(z)$ are not biorthogonal filters, the frame-based pyramid reconstruction (4) doesn't satisfy the PR condition. Thus, its performance would be suffered. However, the

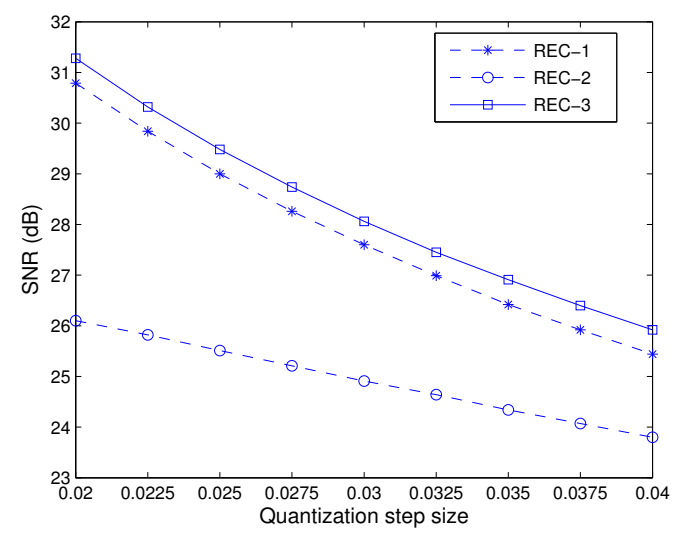

Fig. 5. Comparisons of reconstructions from the quantized LP coefficients of $512 \times 512$ 'Barbara' image. The LP is decomposed with two levels, and $H$ and $G$ filters are the lowpass filter listed in Table 1.

LP reconstruction (12) always satisfies the PR condition, and can still maintain good performance when $H(z)$ and $G(z)$ are lowpass filters. For the simplicity, let REC-1 denote the usual reconstruction (2), REC-2 denote the frame-based pyramid reconstruction (4), and REC-3 denote the special lifting reconstruction (12). Considering the same examples in [4], we compare the performance of these three LP reconstruction schemes when $H(z)$ and $G(z)$ are the same lowpass filter given in Table 1 and $M=2$.

Firstly, we consider an image coding application where uniform scalar quantizers with equal step sizes for the LP coefficients are applied (in open-loop mode [4]). Fig. 5 draws the SNR result for the 'Barbara' image of size $512 \times 512$. It demonstrates that REC-3 has $0.5 \mathrm{~dB}$ gain than REC-1, while REC- 2 has around $2.5 \mathrm{~dB}$ worse than REC-1. Secondly, we consider a typical denoising application where the LP coefficients are usually thresholded so that only the $m$ most significant coefficients are retained. Table 2 lists the numerical denoising results for three standard test images. We observe that REC-3 consistently gives better performance by around $0.4 \mathrm{~dB}$ in SNR than REC-1. While REC-2 has worse performance than REC1 since the PR property is not satisfied. Note that when the LP filters are biorthogonal, e.g., 9/7 biorthogonal wavelet filters, REC-3 has exactly the same performance as REC-2, which can provide better performance than REC-1 by around $0.5 \mathrm{~dB}$ in SNR as presented in [4]. However, the biorthogonal filters could introduce annoying aliasing components into low-resolution LP subbands, especially in image texture and/or edges regions. While the lowpass filter can generate more pleasing visual quality [6].

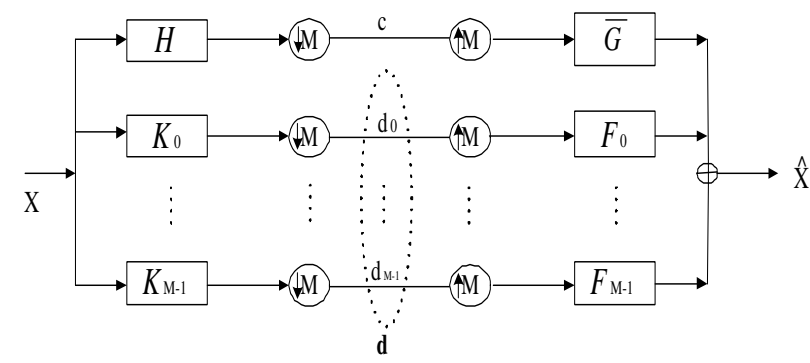

Fig. 6. Laplacian pyramid as an oversampled FB, where $\left\{d_{0}, d_{2}, \ldots, d_{M-1}\right\}$ are the polyphase components of $d[n][4]$. 
Table 2. SNR values of the reconstructed signals from the $m$ most significant LP coefficients. The image sizes are 512x512, and the LP is decomposed with six levels.

\begin{tabular}{|c|c|ccc|}
\hline \multicolumn{2}{|c|}{$m$} & $2^{12}$ & $2^{14}$ & $2^{16}$ \\
\hline \multirow{3}{*}{ Barbara } & REC-1 & 9.43 & 12.60 & 21.55 \\
& REC-2 & 8.80 & 11.38 & 15.67 \\
& REC-3 & $\mathbf{9 . 6 3}$ & $\mathbf{1 2 . 9 6}$ & $\mathbf{2 2 . 2 2}$ \\
\hline \multirow{3}{*}{ Goldhill } & REC-1 & 11.28 & 15.44 & 23.04 \\
& REC-2 & 10.27 & 12.72 & 15.20 \\
& REC-3 & $\mathbf{1 1 . 6 9}$ & $\mathbf{1 5 . 6 8}$ & $\mathbf{2 3 . 7 0}$ \\
\hline \multirow{3}{*}{ Peppers } & REC-1 & 13.37 & 18.80 & 25.78 \\
& REC-2 & 11.43 & 14.03 & 15.31 \\
& REC-3 & $\mathbf{1 3 . 7 5}$ & $\mathbf{1 9 . 3 5}$ & $\mathbf{2 6 . 2 9}$ \\
\hline
\end{tabular}

\subsection{Multilevel Laplacian Pyramid}

Given the polyphase analysis matrix (1) and reconstruction matrix (12), the LP can be expressed as an oversampled FB shown in Fig. 6. The equivalent filters in Fig. 6 can be formulated as

$$
\begin{aligned}
K_{i}(z) & =z^{i}-G_{i}\left(z^{M}\right) H(z), \text { for } i=0, \ldots, M-1,(13) \\
\bar{G}(z) & =T\left(z^{M}\right) G(z), \\
F_{i}(z) & =z^{-i}-G(z) H_{i}\left(z^{M}\right), \text { for } i=0, \ldots, M-1 .(15)
\end{aligned}
$$

Given lowpass filters $G(z)$ and $H(z)$, it is easy to see that $\bar{G}(z)$ is still a lowpass filter, and $K_{i}(z), F_{i}(z)$ are all high-pass filters.

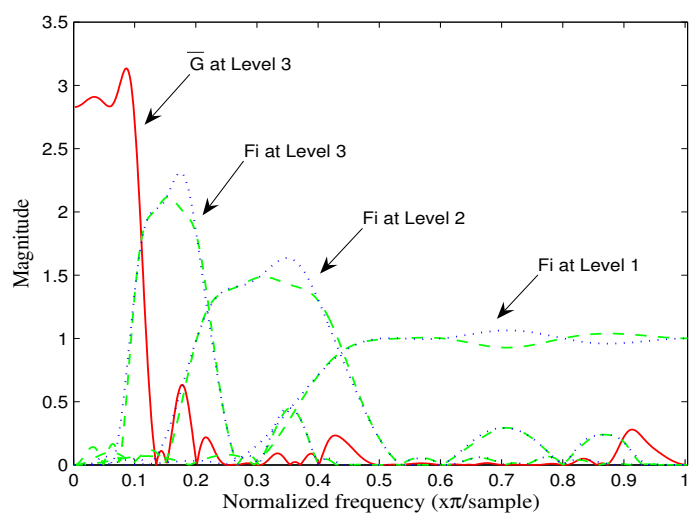

Fig. 7. Frequency responses of the equivalent filters for 3-level LP reconstruction (12).

The multilevel representation can be achieved when the LP scheme is iterated on the coarse signal $c[n]$. For the LP reconstruction (12), the equivalent synthesis filters at the $n$-level LP reconstruction are

$$
\bar{G}_{i}^{(n)}(z)=\prod_{j=0}^{j=n-1} \bar{G}\left(z^{M^{j}}\right),
$$

$F_{i}^{(n)}(z)=F_{i}\left(z^{M^{n-1}}\right) \prod_{j=0}^{j=n-2} \bar{G}\left(z^{M^{j}}\right)$, for $i=0, \ldots, M-1$.

Fig. 7 shows an example of frequency responses of the equivalent filters (17) when the LP filters are the lowpass filter from Table 1. It depicts that the synthesis filters are bandpass and match with the frequency response regions of corresponding subbands. Thus, the
REC-3 reconstruction scheme can confine the errors from highpass subbands of a multilevel LP. This leads to the better performance than REC-1 in coding applications. It also has the prominent advantage over REC-1 when the errors in the LP coefficients have nonzero mean. In such case, with the REC-1 reconstruction, the nonzero mean propagates through all lowpass synthesis filters and appears in the reconstructed signal. In the contrast, with REC-3 reconstruction, the nonzero mean is cancelled by the bandpass filters. We consider the same example in [4], that's, the errors in the LP coefficients (6 levels LP decompositions) are uniformly distributed in $[0,0.1]$. The SNR values for three reconstruction schemes REC-1, REC-2, and REC-3 are $6.25 \mathrm{~dB}, 14.17 \mathrm{~dB}$ and $17.20 \mathrm{~dB}$, respectively. Although synthesis functions of REC-3 have similar frequency responses to those of REC-2, it has better noise elimination performance because REC-2 does not satisfy the PR condition for the given lowpass filter.

\section{CONCLUSION}

In this paper, we present a generic lifting-based LP reconstruction algorithm to characterize all synthesis FBs satisfying the PR property. For the LP with decimation factor $M$, our generic representation only has $M$ free parameters in constat to $M^{2}+M$ free entries in the generic reconstruction FBs presented in [4]. This leads to considerable simplifications in optimum FB design. Two typical lifting-based reconstruction schemes are derived from our general reconstruction algorithm. The first scheme presents the general solutions of dual frame reconstruction. It can minimize white noise errors in LP coefficients, while usually invokes IIR filters. The second special scheme allows to choose lowpass filters to suppress aliasing in low resolution LP subbands efficiently and at the same time lead to improvements over the usual LP method in the presence of noise. This new lifting LP reconstruction can improve H.264 spatially scalable video coding performance and the results will be presented in our other papers.

\section{REFERENCES}

[1] P. J. Burt and E. H. Adelson, "The Laplacian pyramid as a compact image code," IEEE Trans. Commun., vol. COM-31, pp. 532-540, Apr. 1983.

[2] J. Kovacevic and A. Chebira, "Life beyond bases: The advent of frames," IEEE Signal Processing Mag., 2007.

[3] H. Schwarz, D. Marpe, and T. Wiegand, "Overview of the scalable extension of the H.264/MPEG-4 AVC video coding standard," IEEE Trans. Circuits Syst. Video Tech., Sept. 2007.

[4] M. N. Do and M. Veterli, "Frame pyramid," IEEE Trans. Signal Processing, vol. 51, no. 9, pp. 2329-2342, Sept. 2003.

[5] Z. Cvetkovic and M. Vetterli, "Oversampled filter banks," IEEE Trans. Signal Processing, vol. 46, no. 5, pp. 1245-1255, May 1998.

[6] M. Flierl and P. Vandergheynst, "An improved pyramid for spatially scalable video coding," in Proc. IEEE International Conference on Image Processing, Genova, Italy, Sept. 11-14 2005, vol. 2 , pp. $878-881$.

[7] L. Liu, L. Gan, and T. D. Tran, "General reconstruction of laplacian pyramid and its dual frame solutions," in 41 st Conf. on Info. Sci. and Sys., Baltimore, MD, March 2007.

[8] L. Gan and C. Ling, "Computation of the dual frame:forward and backward Greville formulas," in Proc. IEEE Int. CASSP, 2007. 